\title{
The time has come to move from coronary angiography to physiological assessment of coronary lesions
}

Josef Veselka1,2

1Department of Cardiology, University Hospital Motol, Prague, Czech Republic $2^{\text {2nd }}$ Medical School, Charles University, Prague, Czech Republic

Submitted: 29 May 2012

Accepted: 28 June 2012

Arch Med Sci 2013; 9, 1: 1-2

DOI: 10.5114/aoms.2013.33342

Copyright @ 2013 Termedia \& Banach

In the past, revascularization for acute coronary syndromes was demonstrated to be a life-saving procedure [1]. Conversely, the efficacy of percutaneous coronary interventions $(\mathrm{PCl})$ in patients with stable angina pectoris has been a matter of ongoing debate. Large randomized trials comparing revascularization with optimal medical therapy failed to demonstrate the benefit of the interventional approach [2]. Therefore, $\mathrm{PCI}$ has been performed mainly in patients with objective evidence of ischemia unresponsive to medical therapy.

In clinical practice, we mostly rely on the angiographic appearance of coronary lesions, and probably the majority of patients do not undergo stress testing prior to $\mathrm{PCl}[3,4]$. Based on this statement, one would presume that i) coronary angiography is the optimal method for evaluation of coronary artery disease; ii) coronary angiography demonstrates not only the coronary anatomy, but also the hemodynamic consequences of possible atherosclerotic lesions; iii) interventional therapy based on coronary angiography in stable patients is not only safe but effective in the longterm follow-up. Unfortunately, answers to these questions have been widely known for years and given a resounding NO.

In the past, two reliable methods leading to improvements in the accuracy of coronary angiography have been introduced. Interestingly, we demonstrated the first practical experience with intracoronary ultrasound more than a decade ago [5]. Since that time, virtual histology and optical coherence tomography have significantly improved our options for evaluation of coronary anatomy. Moreover, several studies have demonstrated that intravascular ultrasound guided interventions resulted in a reduced incidence of adverse clinical outcomes, especially in patients with proximal stenoses (left main coronary artery or proximal bifurcation lesions) [6]. On the other hand, evaluation of coronary anatomy and accurate morphology of coronary lesions have certain limitations, both with regard to hemodynamic significance and assessing the extent of ischemic myocardium, which is probably the key to choosing an optimal therapy. The only way currently to easily establish the hemodynamic significance of a borderline coronary lesion is a fractional flow reserve (FFR) measurement, which identifies ischemia-causing coronary stenoses with an accuracy of $>90 \%$ [7]. Fractional flow reserve is defined as the ratio between distal coronary pressure and aortic pressure, both measured simultaneously at maximal hyperemia, induced usually with adenosine. Moreover, an FFR $\leq 0.8$ is reliably associated with significant coronary ischemia and has adverse clinical consequences [7]. Recently, the relationship between intravascu-

\author{
Corresponding author: \\ Josef Veselka MD, PhD \\ Department of Cardiology \\ $2^{\text {nd }}$ Medical School \\ Charles University \\ University Hospital Motol \\ V úvalu 84, Praha 5, 15000 \\ Czech Republic \\ E-mail: \\ josef.veselka@fnmotol.cz
}


lar ultrasound and FFR measurements has changed, based on elegant studies published by Korean researchers $[8,9]$. They re-evaluated the older intravascular criteria for functionally significant lesions, demonstrating that the best cut-off values predicting pathological results of FFR were minimal luminal areas of $2.4 \mathrm{~mm}^{2}$ for proximal coronary lesions in the left anterior descending artery, $1.6 \mathrm{~mm}^{2}$ for the left circumflex artery and $2.4 \mathrm{~mm}^{2}$ for the right coronary artery. Apparently, these new morphological criteria are far stricter than the older $\left(4 \mathrm{~mm}^{2}\right)$ guideline. Interestingly, this finding clearly explained the old clinical experience of many interventional cardiologists ("if you want to dilate, use intravascular ultrasound prior to intervention - if you do not, use FFR examination").

The most important reason for daily use of FFR in catheterization laboratories is the fact that coronary angiography is not accurate in determining the functional severity of coronary lesions [7]. It has been shown that coronary angiography may either over- or underestimate the severity of coronary stenoses. This "visual-functional" mismatch was nicely demonstrated in a post-hoc analysis of the FAME study [7]. In patients with angiographically established diagnosis of triple vessel disease, only $14 \%$ had real triple vessel disease, according to FFR, whereas $9 \%$ of the patients had no hemodynamically significant stenoses at all. Of 1329 evaluated lesions with angiographic stenoses greater than $50 \%$, only $61 \%$ had FFR $\leq 0.8$. Similarly, in patients with angiographic diagnosis of multivessel disease, only $46 \%$ had real multivessel coronary artery disease. It is notable that the optimal therapy for these patients is still debatable, because of the lack of correctly designed, randomized trials, reflecting the rapid development of interventional cardiology in the drug-eluting stent era. Recently, the FAME 2 study presented at the PCR Congress in Paris (May 2012) demonstrated in a randomized trial design that FFR measurements can identify patients with stable angina pectoris who require not only optimal medical therapy, but also PCI [10]. Therefore, FFR might be the key factor in identifying stable angina patients requiring revascularization. It is of note that in most previous trials dealing with stable angina patients, the diagnosis of coronary artery disease was based only on the angiographic appearance of the coronary arteries. Therefore, it seems probable that a sizeable proportion of patients without significant ischemia were included in these trials, which markedly influenced their results.

So, how should these data be translated into daily clinical practice? Probably a significant proportion of patients with angiographically established triple vessel coronary artery disease, who should theoretically be treated by coronary artery bypasses, do not suffer from such diffuse coronary atherosclerosis and could be safely treated with per- cutaneous revascularization. Moreover, some of them do not need revascularization at all. On the other hand, there are some patients with much more pronounced coronary atherosclerosis than is demonstrated by coronary angiogram, and these patients should be treated by cardiac surgeons. Therefore, at present and in the near future, we should overcome the "visual-functional" mismatch and most angiographic stenoses should be assessed by FFR. The time has come.

\section{Acknowledgments}

Supported by the project for conceptual development of research organization 00064203 (Ministry of Health, Czech Republic).

\section{References}

1. Athyros VG, Gossios TD, Tziomalos K, Florentin M, Karagiannis A, Mikhailidis DP. Is there an additional benefit from coronary revascularization in diabetic patients with acute coronary syndromes or stable angina who are already on optimal medical treatment? Arch Med Sci 2011; 7: 1067-75.

2. Brooks MM, Boden WE, Frye RL. Clinical implications of the BARI 2D and Courage trials: overview. Coron Artery Dis 2010; 21: 383-5.

3. Veselka J, Hájek P, Malý M, et al. Predictors of coronary intervention-related myocardial infarction in stable angina patients pre-treated with statins. Arch Med Sci 2011; 7: 67-72.

4. Yu DQ, Lin SG, Chen JY, et al. Effect of atorvastatin therapy on borderline vulnerable lesions in patients with acute coronary syndrome. Arch Med Sci 2011; 7: 433-9.

5. Veselka J. Intracoronary ultrasound: the "platinum standard" in evaluating morphology of coronary stenoses. Vnitr Lek 2000; 46: 480-3.

6. Parise H, Maehara A, Stone GW, Leon MB, Mintz GS. Meta-analysis of randomized studies comparing intravascular ultrasound versus angiographic guidance of percutaneous coronary intervention in pre-drug-eluting stent era. Am J Cardiol 2011; 107: 374-82.

7. Tonino PAL, Fearon WF, De Bruyne B, et al. Angiographic versus functional severity of coronary artery stenoses in the FAME Study: fractional flow reserve versus angiography in multivessel evaluation. J Am Coll Cardiol 2010; 55: 2816-21.

8. Koh JS, Koo BK, Kim JH, et al. Relationship between fractional flow reserve and angiographic and intravascular ultrasound parameters in ostial lesions. J Am Coll Cardiol Int 2012; 5: 409-15.

9. Koo BK, Yang HM, Doh JH, et al. Optimal intravascular ultrasound criteria and their accuracy for defining the functional significance of intermediate coronary stenoses at different locations. J Am Coll Cardiol Int 2011; 4: 803-11.

10. De Bruyne B. FFR-guided PCI plus optimal medical treatment versus optimal medical treatment alone in patients with stable coronary artery disease (FAME 2 trial): preliminary results of cohort A. EuroPCR 2012, May 15. Paris, France. 